\title{
Cell type identification from single-cell transcriptomes in melanoma
}

\author{
Qiuyan Huo, Yu Yin, Fangfang Liu, Yuying Ma, Liming Wang ${ }^{*}$ and Guimin Qin*
}

From The International Conference on Data Science, Analytics, and Engineering (IDSAE) 2020/2021

Virtual. 24-25 January 2021

\begin{abstract}
Background: Single-cell sequencing approaches allow gene expression to be measured at the single-cell level, providing opportunities and challenges to study the aetiology of complex diseases, including cancer.

Methods: Based on single-cell gene and IncRNA expression levels, we proposed a computational framework for cell type identification that fully considers cell dropout characteristics. First, we defined the dropout features of the cells and identified the dropout clusters. Second, we constructed a differential co-expression network and identified differential modules. Finally, we identified cell types based on the differential modules.

Results: The method was applied to single-cell melanoma data, and eight cell types were identified. Enrichment analysis of the candidate cell marker genes for the two key cell types showed that both key cell types were closely related to the physiological activities of the major histocompatibility complex (MHC); one key cell type was associated with mitosis-related activities, and the other with pathways related to ten diseases.

Conclusions: Through identification and analysis of key melanoma-related cell types, we explored the molecular mechanism of melanoma, providing insight into melanoma research. Moreover, the candidate cell markers for the two key cell types are potential therapeutic targets for melanoma.
\end{abstract}

Keywords: Single-cell sequencing, Melanoma, Cell type, Cell marker, IncRNA

\section{Background}

Melanoma is a malignant tumor that develops from melanocytes and is considered a multifactorial disease caused by the interaction between genetic susceptibility factors and environmental exposure $[1,2]$. Although the incidence of many cancers is declining, the incidence of melanoma is increasing $[3,4]$. The prognosis of melanoma is proportionate to the depth of the tumor, which increases with time; thus, melanoma must be identified, detected, and treated in a timely manner [1]. Schomberg

*Correspondence: wanglm@mail.xidian.edu.cn; gmqin@mail.xidian.edu.cn School of Computer Science and Technology, Xidian University, Xi'an 710071, China et al. [5] used RNA sequencing (RNA-seq) to profile luteolin-induced differentially expressed genes (DEGs) in 4 melanoma cell lines and found that luteolin-mediated growth inhibition may be mediated in melanoma cells through simultaneous action on multiple pathways. Mahata [6] proposed a clustering method to explore the subtypes of melanoma and breast cancer. Klinke et al. [7] developed an unsupervised feature extraction and selection strategy to capture functional plasticity separately tailored to breast cancer and melanoma.

The limitations of bulk RNA-seq data are that the molecular expression in a single cell is masked, and the cell heterogeneity in a sample is ignored. With the development of single-cell RNA sequencing 
(scRNA-seq) technology, scRNA-seq data analysis has been widely used in the study of different biological tissues, revealing the meanings of differential gene expression between cells [8-10] and researchers have begun to decipher the functional states of cancer cells at the single-cell level [11-14]. Various methods related to the life sciences have been applied in cancer research and have led to discoveries in cancer evolution, metastasis, treatment resistance and the tumor microenvironment $[15,16]$.

Compared with next generation RNA-seq data, there are more noise data and more dropouts in scRNAseq data. There are several reasons for the dropout phenomenon [17]. Firstly, transcripts do not exist, so zero is an accurate representation of the state of a cell; secondly, the depth of sequencing is low, despite the existence of transcripts, it has not been reported. Thirdly, as part of library preparation, transcripts were not captured or failed to amplify. Some methods were proposed for imputing zeros. Lin et al. [18] introduced the Clustering through Imputation and Dimensionality Reduction(CIDR), which used a novel but very simple implicit imputation approach in a principled way in order to mitigate the impact of dropout values in scRNA-seq data. van Dijk et al. [19] developed the Markov affinity-based graph imputation of cells(MAGIC), to share information between similar cells through data diffusion to denoise the cell count matrix and fill in missing transcripts. Li et al. [20] introduced the scImpute to impute the dropout values in scRNA-seq data. Instead of eliminating the influence of dropout values to improve clustering accuracy, we attempted to amplify the influence of dropout values to explore the molecular mechanisms of melanoma.

In this study, we fully considered the characteristics of scRNA-seq data and identified a variety of cell types in melanoma cancer cells and a series of candidate cell markers for various cell types based on scRNA-seq gene and lncRNA expression data. Furthermore, by evaluation of each cell type, we identified and analyzed the key cell types associated with melanoma and then revealed the pathogenesis of melanoma, providing new insight into its diagnosis and prognosis.

\section{Methods}

In this paper, considering the characteristics of scRNAseq data, we proposed a framework for cell type identification (Fig. 1). The framework consists of three parts: identification of dropout clusters, construction of the differential co-expression network, and identification of cell types and candidate cell markers.

\section{Molecular expression datasets}

The expression profiles used in this experiment were extracted from the EXP0072 dataset in CancerSEA (http://biocc.hrbmu.edu.cn/CancerSEA/goDownload) [21], which was collated from the expression files from the GEO dataset GSE81383 [22]. scRNA-seq was applied to profile the transcriptomes of 307 single cells cultured from three biopsies of three different patients, who had BRAF/NRAS wild type, BRAF mutant/NRAS wild type and BRAF wild/NRAS mutant type metastatic melanoma. The expression profiles contain the expression values for 18,938 protein-coding genes and 15,626 lncRNAs.

\section{Known melanoma-related biomolecules}

To analyze the correlation between each cell type pair, we collated known melanoma-related biomolecules from multiple public databases and published research results.

From the OMIM catalogue (https://omim.org/) [23], the COSMIC database (https://cancer.sanger.ac.uk/ cosmic) [24] and a published study by Bailey et al. [25], we obtained 539, 63 and 24 known melanoma-related genes, respectively. A total of 580 known melanomarelated genes were obtained.

From the Lnc2Cancer 2.0 (http://www.bio-bigdata. net/lnc2cancer/) [26], LncRNADisease v2.0 (http:// www.rnanut.net/lncrnadisease/) [27] and NONCODE v5.0 (http://www.noncode.org/) [28] data-bases, we obtained 24, 2,712 and 14 known melanoma-related lncRNAs, respectively. A total of 2,719 known melanoma-related lncRNAs were obtained.

In addition, we obtained a cell marker entity associated with melanoma from the CellMarker (http://biocc. hrbmu.edu.cn/CellMarker/) [29] database. This entry comprises data from cancer cells in peripheral blood and contains four cell marker genes, MITF, MLANA, PMEL and TYR. Schelker et al. [30] used scRNA-seq data to identify nine main cell types, and the four above mentioned genes (MITF, MLANA, PMEL and TYR) were used as cell markers for melanoma cell types.

\section{Data preprocessing}

We used the EXP0072 dataset in CancerSEA [24], which contains the expression profiles of 18,938 protein-coding genes and 15,626 lncRNAs from 307 melanoma cancer cells.

First, we filtered the genes and lncRNAs with expression values of greater than 0 in fewer than 3 cells or average normalized expression values of less than $10^{-5}$, fitted the normal distribution to the genes (or lncRNAs) and cells, and removed cells in which significantly 


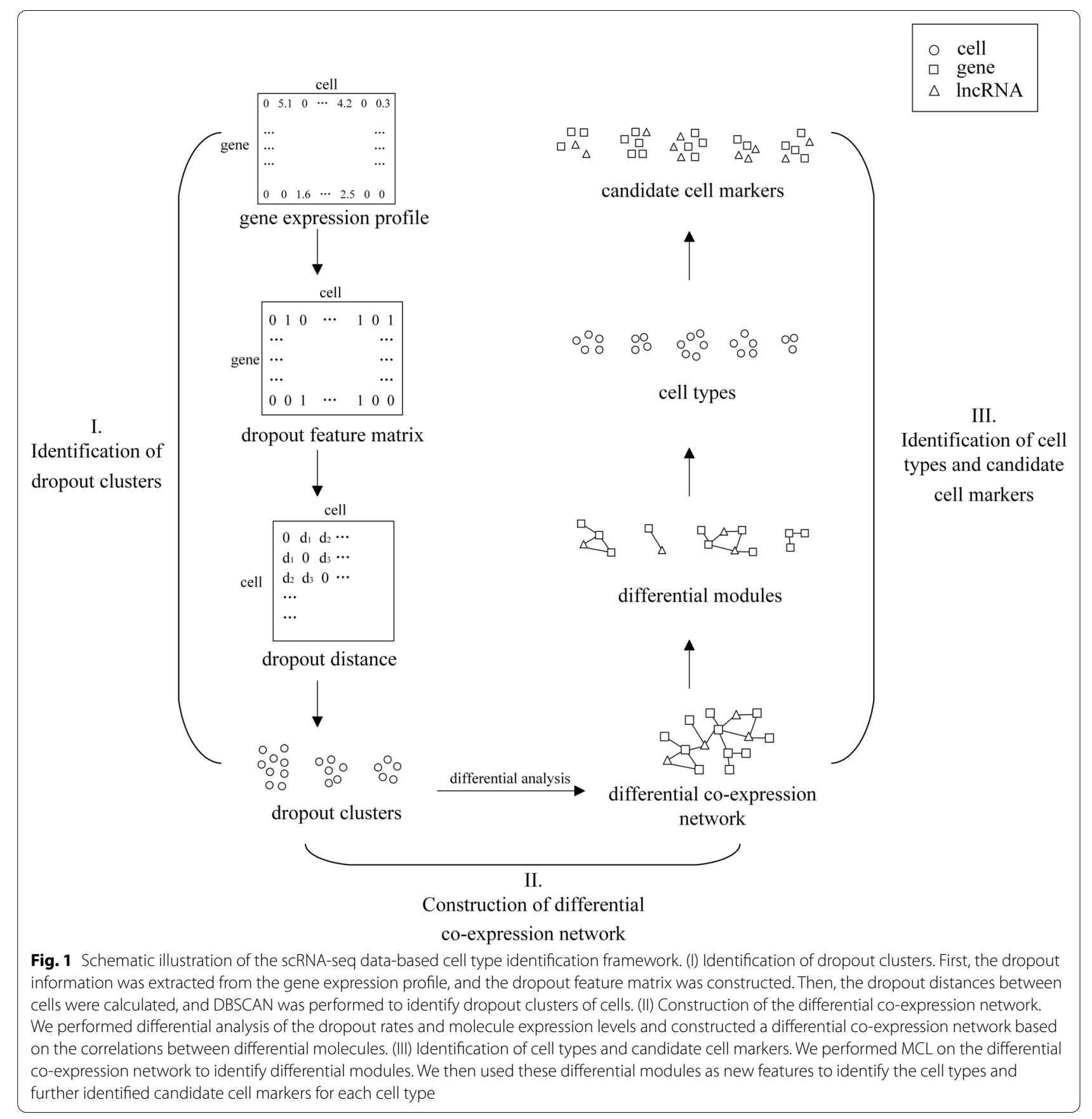

few genes were detected (FDR $<0.05)$. The resulting expression profile consisted of 307 cells, 15,488 genes and 8,524 lncRNAs.

Next, feature selection was performed for the genes and lncRNAs with non-log-transformed expression data using the M3Drop method in the M3Drop package [31].

\section{Feature selection}

Cell clustering is dependent on the selection of genes, and traditional methods generally use the most variable genes as features. An important characteristic of scRNA-seq data is the high dropout rate, which usually accounts for more than half of the values in the 
expression matrix, and M3Drop [31] selects genes based on the dropout property.

M3Drop is based on the Michaelis-Menten equation, which is used to represent enzymatic reactions to fit the relationship between the average expression value and the dropout rate, as shown in (1):

$$
P_{\text {dropout }}=1-S /(K+S)
$$

where $S$ is the average expression level of the gene in all cells, $K$ is the Michaelis constant, and $P_{\text {dropout }}$ is the ratio of the dropout value of the gene to the expression of the gene in all cells, i.e., the dropout rate of the gene.

The parameter $K$ in the Michaelis-Menten equation was used to calculate the specific $K_{j}$ for gene j, and the global KM of all genes was fitted by the $\mathrm{z}$-test. Then, the significant genes were selected as the result of feature selection by multiple testing corrections. The equation for calculating $K_{j}$ is shown as (2):

$$
K_{j}=\left(P_{j} * S_{j}\right) /\left(1-P_{j}\right)
$$

where $P_{j}, S_{j}$ and $K_{j}$ are the corresponding $P_{\text {dropout }}, S$ and $K$ values for gene $j$ in (1).

Identification of dropout clusters.

To fully explore the dropout information in the single-cell expression data, we fuzzed specific gene expression values and highlighted dropout values. According to whether the gene expression in the cell was a dropout value, we binarized the gene expression data. In detail, all expression values greater than 0 in the gene expression profile were recorded as 1 ; otherwise, as 0 . The matrix generated by binarizing the gene expression values was called the dropout feature matrix.

Next, we defined the dropout distance between cells based on the dropout features. There are a large number of zeros in scRNA-seq data, so we applied Manhat$\tan$ distance to measure the distance between cells to avoid bias. Firstly, we calculated the Manhattan distance between each cell pair and then used the z-score to normalize the Manhattan distance to obtain the dropout distance between the cells.

Then, based on the dropout distance between the cells, we clustered cells with density-based spatial application of applications with noise (DBSCAN) [32], which can identify clusters with various shapes and sizes and effectively identify noise in cells. Before clustering, we visualized the data distribution and found it was based on the density distribution, and then compared to some other clustering algorithms(for example, SC3 and pcaReduce), DBSCAN is sensitive to noisy data while SC3 and pcaReduce often mistake noise for true structure, which means DBSCAN is more appropriate for our dataset[17]. We then defined dropout clusters as cell clusters obtained by cluster analysis based on the dropout distance.

Two hyperparameters should be determined in DBSCAN, one is the field radius eps that defines the field range, and the other is the minimum field point MinPts required for the sample to be defined as the core point. The parameter selection method is as follows:

Step 1 initialize MinPts as $\mathrm{M}_{\mathrm{i}}$ and calculate the $\mathrm{M}_{\mathrm{i}}$ distance range $\mathrm{R}$ for all samples. Given $\mathrm{R}$ and step size, say 0.001 , calculate eps and the number of clusters k, and retain the eps that maximizes the silhouette coefficient [33] and set it as $\mathrm{e}_{\mathrm{i}}$.

Step 2 assign $\mathrm{e}_{\mathrm{i}}$ to eps and calculated the maximized the silhouette coefficient [33] of MinPts, marked as $\mathrm{M}_{\mathrm{i}}$.

Step 3 update $M_{i}$ and repeat Step 1 and Step 2. The parameters that maximized the silhouette coefficient[33] are set to be the input of DBSCAN.

\section{Construction of the differential co-expression network}

To analyze the differences among dropout clusters, for each dropout cluster, we divided all the cells into two groups-cells belonging to the cluster and the remaining cells-and performed differential analysis of genes and lncRNAs from two aspects: the dropout rate and molecule expression value.

Differential dropout analysis. We calculated the dropout rate for all gene/lncRNA expression values for each cell group. The genes and lncRNAs with a difference in the dropout rate of greater than $50 \%$ between the two groups of cells were defined as differentially dropout genes (DDGs) and differentially dropout lncRNAs (DDLRs).

Differential expression analysis. We calculated the fold changes in the gene/lncRNA expression values between the two groups of cells and selected genes and lncRNAs with a fold change of greater than two $(|\log F C|>1, p$ value $<0.05)$. Then, we defined these genes and lncRNAs as differentially expressed genes (DEGs) and differentially expressed lncRNAs (DELRs).

Herein, we refer to DDGs and DEGs as differential genes, to DDLRs and DELRs as differential lncRNAs, and to the collective set of differential genes and differential lncRNAs as differential molecules.

Then, we calculated the Spearman correlation coefficient (SCC) between each pair of differential molecules and selected strong correlations with $|\mathrm{SCC}|>0.4$ and $p$ value $<0.05$. The differential molecules with strong correlations constituted the differential co-expression network. The absolute values of the Spearman correlation coefficients were used as the edge weights. 


\section{Identification of cell types and candidate cell markers}

We used the Markov clustering algorithm (MCL) [34] to cluster the differential co-expression network and identify molecule modules, which we call differential modules herein. MCL is a graph-based, rapidly scalable unsupervised clustering algorithm, and it simulates a random flow to discover the communities in the network. Then, we calculated the average expression value of every differential module as a new feature of the cells. Herein, we call this value the differential module feature of cells.

According to the differential module features, we calculated the Manhattan distance between cell pairs and normalized it by the z-score. Then, we applied DBSCAN to cluster the cells, and each cluster was considered a cell type.

For each cell type, we calculated the fold change in the expression level of each gene and lncRNA between cells in that cell type and the remaining cells and selected genes and lncRNAs with a significant difference of at least a fourfold change $(|\log \mathrm{FC}|>2, p$ value $<0.05)$ as the candidate cell marker genes and candidate cell marker lncRNAs for that cell type. Herein, the candidate cell marker genes and lncRNAs are collectively referred to as candidate cell markers for a cell type.

\section{Results}

\section{Feature selection}

The results of data preprocessing are shown in Fig. 2(A) and (B). Finally, 3,454 genes and 966 lncRNAs were selected for further analysis. We also performed log transformation on the expression data with a base of 2 and an offset of 1 .

\section{Identification of dropout clusters and differential molecules}

The dropout distances between every pair of cells were calculated according to the dropout feature matrix, and the cells were analyzed with DBSCAN [32]. Four cell dropout clusters that individually contained 27, 8, 51 and 45 cells. The result of the dropout clusters was a transition to get the final cell types.

Furthermore, differential dropout analysis and differential expression analysis were performed on different dropout clusters, and the number of differential

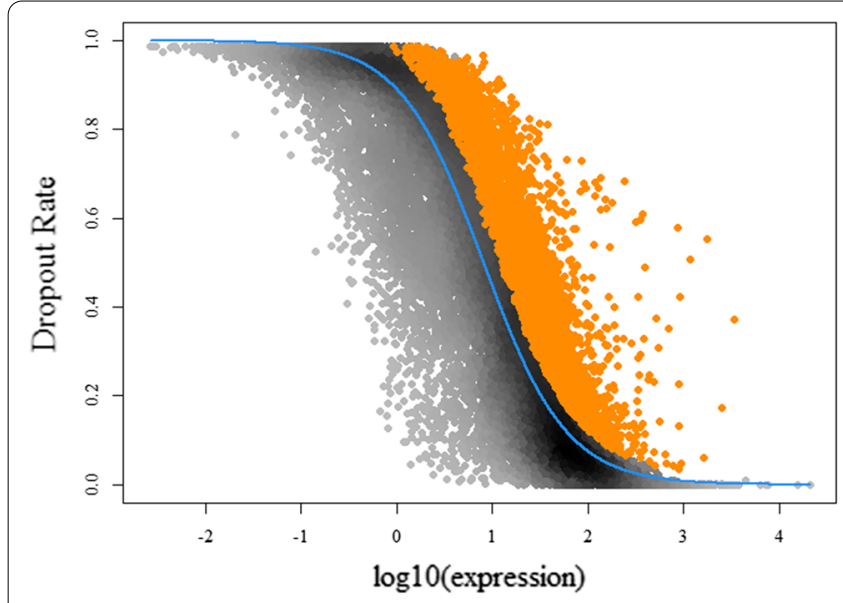

(A)

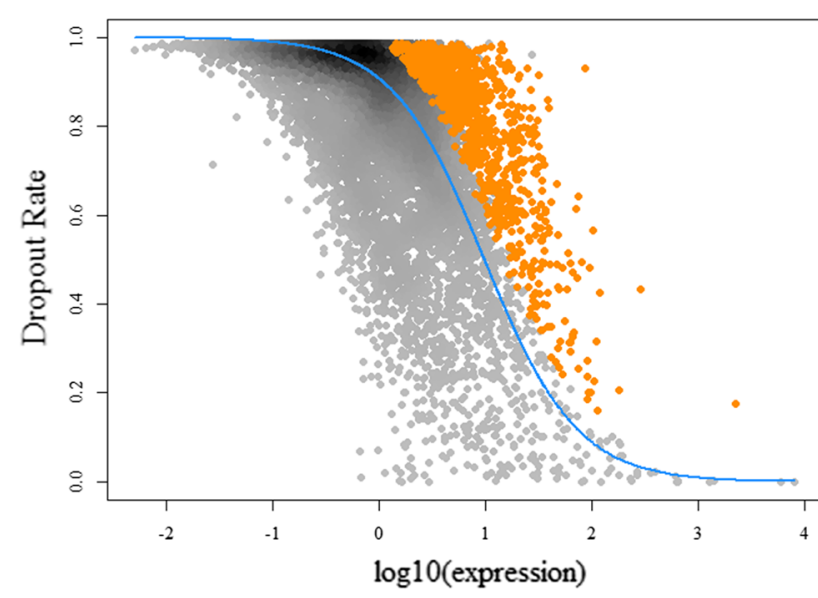

(B)

Fig. 2 Results of feature selection using M3Drop. The solid blue lines indicate the Michaelis-Menten curves fit to all genes or IncRNAs, and the orange dots indicate the significant genes or IncRNAs with an FDR of $<0.01$ in the hypothesis test. A Results of feature selection for genes. B Results of feature selection for IncRNAs

Table 1 Number of differential molecules in the dropout clusters, including the number of differential expressed genes/IncRNAs in each dropout cluster and the number of differential molecules in all dropout clusters

\begin{tabular}{lllllll}
\hline Dropout cluster & & $\mathbf{1}$ & $\mathbf{2}$ & $\mathbf{3}$ & $\mathbf{4}$ & Sum \\
\hline Differential expressed genes & DEGs & 805 & 6 & 1185 & 1067 & 1940 \\
& DDGs & 17 & 46 & 80 & 143 & 146 \\
Differential expressed IncRNAs & DELRs & 5 & 2 & 119 & 7 \\
& DDLRs & 0 & 4 & 7 & 16 \\
\hline
\end{tabular}


molecules identified is shown in Table 1. Since some DEGS, DDGs, DELRs and DDLRs may belong to multiple clusters, Sum represents the total number of four clusters' molecules. More differential expressed genes than differential expressed lncRNAs were identified, and the differences in the expression levels were generally greater than the differences in the dropout rates. Differential analysis of all dropout clusters identified 1950 differential expressed genes and 209 differential expressed lncRNAs. The number 1950 means the total number of DEGs and DELRs after deduplication. Similarly, the number 209 means the total number of DDGs and DDLRs after deduplication.

\section{Analysis of the differential co-expression network}

For the 2159 differential expressed molecules (1950 differential expressed genes and 209 expressed differential lncRNAs) obtained from the differential analysis, the Spearman correlation coefficient between each pair of differential molecules was calculated with cut-off criteria of $|\mathrm{SCC}|>0.4$ and $p$ value $<0.05$. We obtained 48,940 strong correlations among differential expressed molecules, specifically, 17,908 positive correlations and 31,032 negative correlations. The resulting differential co-expression network was an undirected and weighted network consisting of 892 nodes and 48,940 edges.
The differential co-expression network was a scale-free network with a power law node degree distribution. The protein-coding gene HLA-DRA was a hub node in the network, with a degree of 484. GeneCards [35] shows that $H L A-D R A$ is a protein-coding gene whose main function is to bind to peptides produced by antigens in the endocytosis of antigen-presenting cells (APCs) and display them on the cell surface for recognition by $\mathrm{CD} 4+\mathrm{T}$ cells.

The edge weights in the differential co-expression network were calculated as the absolute values of the Spearman correlation coefficients. Among the connected nodes, the protein-coding gene PHACTR1 had the strongest correlation with the lncRNA AL008729.2, with the Spearman correlation coefficient of 0.96 .

\section{Identification of differential modules and cell types}

The differential molecules in the differential co-expression network were further divided by MCL with the inflation parameter set at 2.5. Twenty differential modules were identified. For each identified differential module, we extracted the sub-network from the differential coexpression network (Fig. 3).

Then, we calculated the differential module features of cells and used DBSCAN to identify cell types. A total of eight cell types were identified and are denoted by the letters $\mathrm{A}-\mathrm{H}$. The number of cells of each cell type is shown in Table 2. Cell type B contained significantly more cells

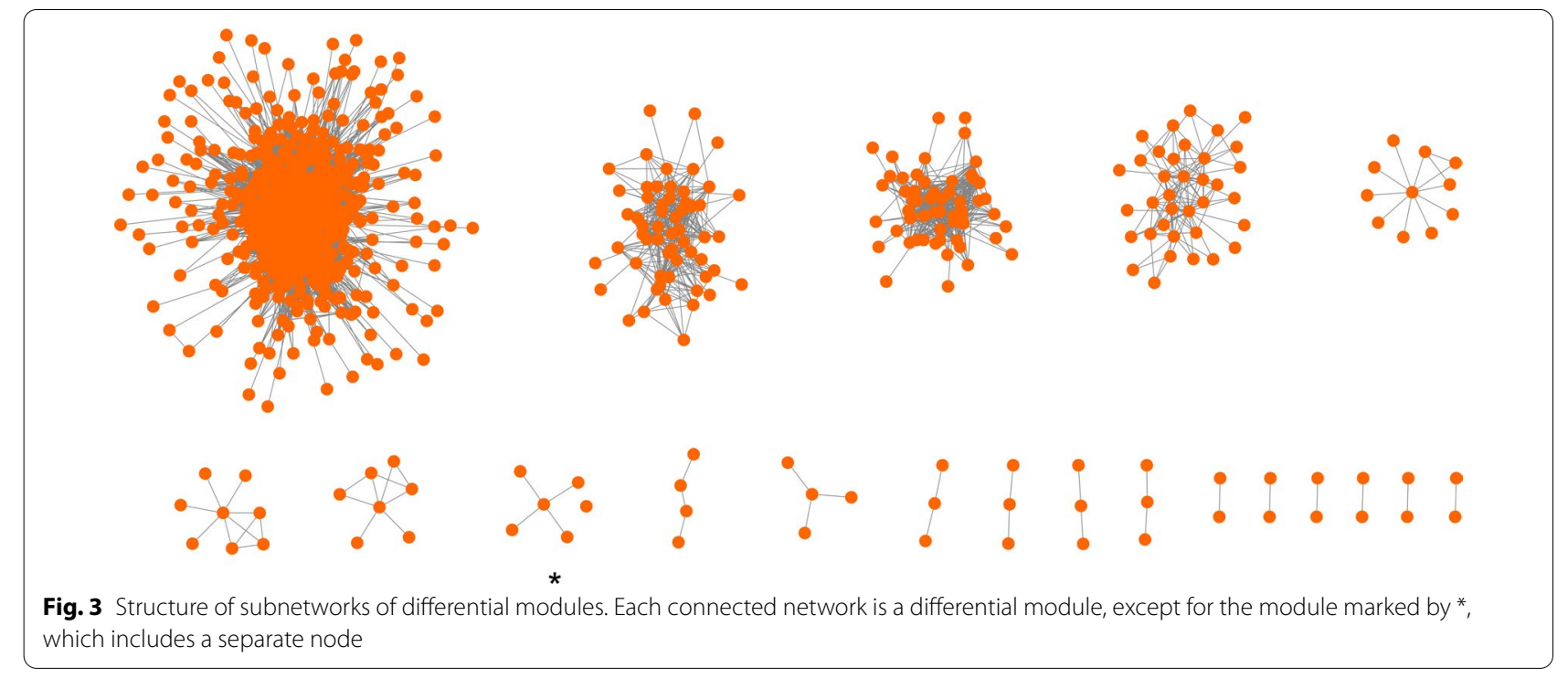

Table 2 Number of cells in each cell type

\begin{tabular}{llllllll}
\hline Cell type & A & B & C & D & E & F & G \\
\hline Number of cells & 10 & 121 & 8 & 7 & 11 & 22 & 22 \\
\hline
\end{tabular}




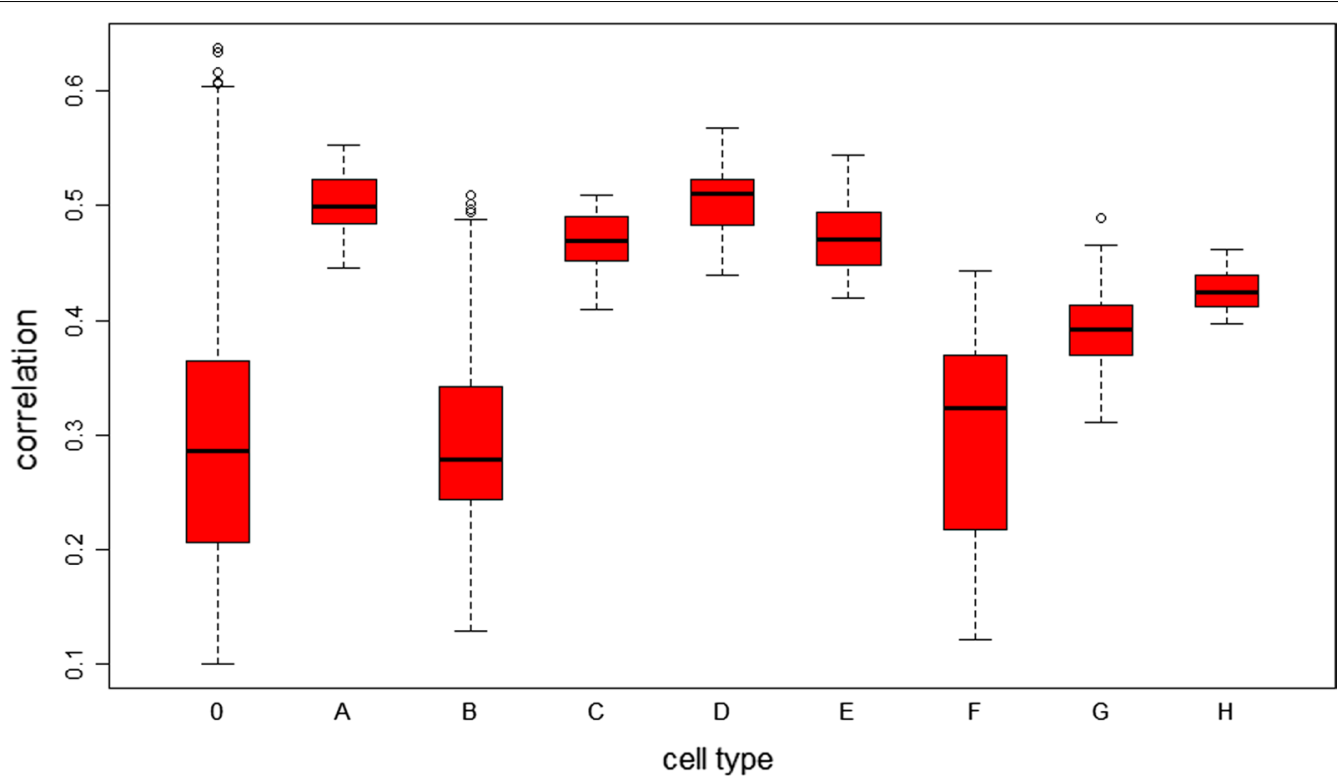

Fig. 4 Similarity of molecular expression patterns in each cell type. The Spearman correlation coefficients for the gene and IncRNA expression levels in all cells in each cell type were calculated, and all correlation coefficients were significant

than the other seven cell types, each of which contained a relatively small number of cells. In addition, 100 cells did not belong to any cell type; we then combined all of these cells into a distinct cell type named cell type 0 .

\section{Analysis of cell similarity in cell types}

The Spearman correlation coefficient between each pair of cells in the same cell type was calculated according to the expression values of genes and lncRNAs to indicate the similarity of the expression patterns between the two cells. The boxplot of the similarity between cells in each cell type is shown in Fig. 4.

These results showed that the expression patterns of any two cells in the same cell type were positively correlated with significant $p$ values. Figure 4 indicates that the average similarity of cells in all cell types except cell type $B$ was significantly higher than that in cell type 0. In particular, in cell types A, C, D, and E, the lowest correlation coefficient between two cells was greater than 0.4 , and the correlation coefficients between all cells in cell types $\mathrm{G}$ and $\mathrm{H}$ were greater than 0.3 . In addition, in cell type 0 , the correlation coefficients between cells had a large span and a low average value, consistent with the experimental results indicating that the cells did not belong to the same cell type.

\section{Differential analysis between cell types}

Furthermore, the R package Limma [36] was used to calculate the fold changes in expression levels, and the significantly differential genes and lncRNAs with at least a fourfold change in expression were selected as candidate cell markers. The number of candidate cell markers obtained are shown in Table 3. More than 200 candidate cell markers were identified for cell types A, E, G, and H, indicating significant differences between cells in these cell types and other cell types.

We analyzed regulation directions of the candidate cell markers for each cell type. In cell types A, C, and D, all candidate cell markers were upregulated $(\log F C>0)$. In cell type E, 3 candidate cell marker genes were downregulated $(\log \mathrm{FC}<0)$, and the remaining candidate cell markers were upregulated. In cell type $F$, only one

Table 3 Number of candidate cell markers for each cell type, including candidate cell marker genes and candidate cell marker IncRNAs

\begin{tabular}{|c|c|c|c|c|c|c|c|c|c|}
\hline Cell type & 0 & A & B & C & D & E & $\mathbf{F}$ & G & $\mathrm{H}$ \\
\hline Number of candidate cell marker genes & 0 & 213 & 79 & 20 & 96 & 207 & 92 & 216 & 224 \\
\hline Number of candidate cell marker IncRNAs & 0 & 23 & 5 & 7 & 15 & 37 & 0 & 6 & 38 \\
\hline Number of candidate cell markers & 0 & 236 & 84 & 27 & 111 & 244 & 92 & 222 & 262 \\
\hline
\end{tabular}


candidate cell marker gene was upregulated, and the rest were downregulated. Candidate cell markers in other cell types were upregulated and downregulated in different patterns.

In addition, in cell type 0 , no significant similarity was observed among the expression patterns of the cells, and no candidate cell markers were found, which verified the reliability of the results for the eight identified cell types.

\section{Prediction of candidate cell markers in melanoma}

To verify the relationships between the cell types and melanoma, we collated 580 known melanoma-related genes and 2719 known melanoma-related lncRNAs from multiple databases and published research results and compared them with candidate cell markers in each cell type (see the Materials and Methods for details). The results are shown in Fig. 5.

Figure 5(A) indicates that except for cell type C, which had only 20 candidate cell marker genes, all cell types had known melanoma-related genes among the candidate cell marker genes. The candidate cell markers for cell types $\mathrm{H}$ and $\mathrm{G}$ included 29 and 28 known melanoma-related genes, respectively. In addition, in cell types $B, G$, and $H$, known melanoma-related
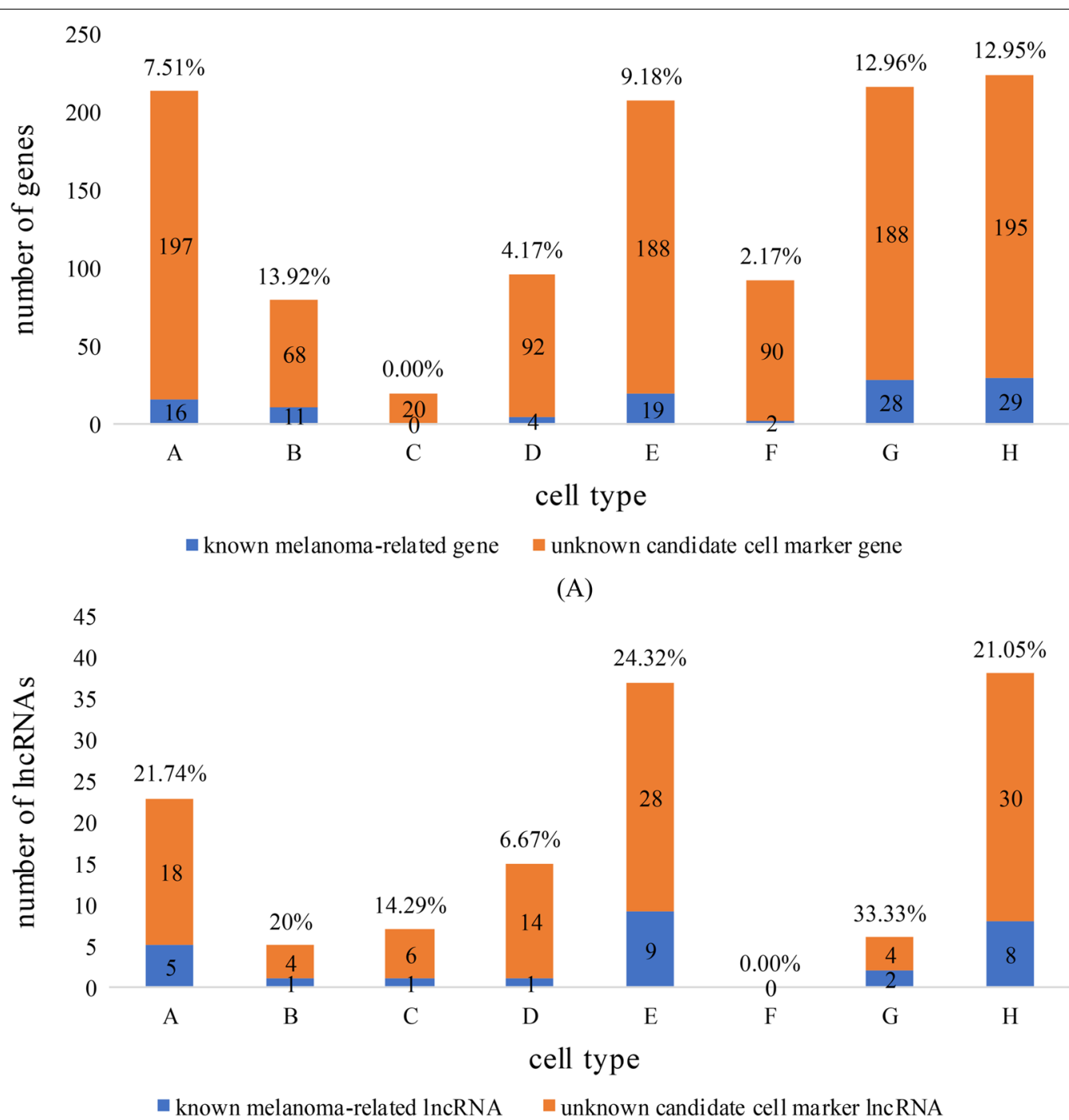

(B)

Fig. 5 Relationships between candidate cell markers for each cell type and known melanoma-related biomolecules. A Relationships between candidate cell marker genes and known melanoma-related genes, including the numbers and percentages of known melanoma-related genes. B Relationships between candidate cell marker IncRNAs and known melanoma-related IncRNAs, including the numbers and percentages of known melanoma-related IncRNAs 
genes accounted for more than $10 \%$ of all candidate cell marker genes.

Figure 5(B) indicates that 9, 8, and 5 candidate cell marker lncRNAs in cell types $\mathrm{E}, \mathrm{H}$, and A, respectively, were known melanoma-related lncRNAs and that known melanoma-related lncRNAs accounted for the largest percentage of candidate cell marker lncRNAs in cell type G (33.33\%).

The above analysis of the correlations between eight cell types and melanoma indicated that candidate cell markers in cell types A, B, E, G, and H, especially cell types $\mathrm{G}$ and $\mathrm{H}$, were strongly related to melanoma.

\section{Analysis of known melanoma cell markers}

Four known melanoma cell marker genes, MITF, MLANA, PMEL and TYR, were obtained from CellMarker [29]. Then, we investigated whether these four known melanoma cell markers appeared among the candidate cell markers for each cell type.

All four known melanoma cell markers were candidate cell marker genes for cell type $\mathrm{H}$, and $M L A N A, P M E L$, and $T Y R$ were candidate cell marker genes for cell type G. In addition, the protein-coding gene $P M E L$ was a candidate cell marker gene for five cell types (all cell types except $\mathrm{C}, \mathrm{D}$, and F). PMEL plays a central role in the biogenesis of melanosomes and participates in the maturation of melanosomes from stage I to stage II [37, 38]. According to GeneCards, PMEL is associated with the incidence of various melanomas, such as skin melanoma, gallbladder melanoma, and melanoma in congenital melanocytic nevus.

$P M E L$, also known as premelanosome protein gene, participates in the maturation process of melanosomes from phase I to phase II, and plays a central role in melanogenesis [37, 38]. MITF plays a role in multiple activity levels that determine the fate of melanoma cells. Melanoma cells that highly express MITF can differentiate or proliferate. Stem cell-like or invasive potential can cause low MITF activity. And long-term MITF inhibition will drive cell senescence [39]. MITF up- or down-regulation modulates MLANA expression in parallel directions at both mRNA and protein levels. As a target gene for melanocyte restriction, MLANA may provide an opportunity to study whether their melanocyte restriction expression is produced by the unique activity of the MITF melanocyte isotype, or whether other transcription factors may contribute (together with $M I T F$ ) give melanocytespecific expression [40]. TYR, TYRP1 and downstream enzymes metabolize tyrosine to melanin [41].

These results showed that the candidate cell markers for cell types $\mathrm{G}$ and $\mathrm{H}$ were closely related to the known melanoma cell markers and that other unknown candidate cell markers in these two cell types could be potential driver biomolecules for melanoma.

\section{Enrichment analysis of key cell types}

The previous analysis of the eight cell types indicated that cell types $\mathrm{G}$ and $\mathrm{H}$ were highly correlated with melanoma and that the cells belonging to these cell types had similar expression patterns and were significantly different from the cells not belonging to these cell types. We defined these two cell types as the key cell types associated with melanoma and further used the $\mathrm{R}$ package clusterProfiler [42] to perform Gene Ontology (GO) functional and Kyoto Encyclopedia of Genes and Genomes (KEGG) pathway enrichment analyses of their candidate cell marker genes to explore the pathogenesis of melanoma.

We performed GO functional enrichment analysis, including analysis of biological process (BP), molecular function (MF) and cellular component (CC) terms, and focused on GO terms with an adjusted p-value (p.adjust) of $<0.01$. We obtained 113 and $95 \mathrm{GO}$ terms related to cell types $\mathrm{G}$ and $\mathrm{H}$, respectively; the top three $\mathrm{GO}$ terms in the BP, MF and CC aspects are shown in Tables 4 and

Table 4 Gene Ontology (GO) functional enrichment analysis of candidate cell marker genes for cell type G. The top 3 GO terms in the biological process (BP), molecular function (MF) and cellular component (CC) aspects are listed (p.adjust < 0.01 )

\begin{tabular}{|c|c|c|c|c|}
\hline Aspect & GO ID & Descriptions & p.adjust & Count \\
\hline & GO:0140014 & Mitotic nuclear division & $7.11 \mathrm{E}-06$ & 17 \\
\hline \multirow[t]{3}{*}{$\mathrm{BP}$} & GO:0048285 & Organelle fission & $2.83 \mathrm{E}-05$ & 20 \\
\hline & GO:0000280 & Nuclear division & $2.83 \mathrm{E}-05$ & 19 \\
\hline & GO:0023026 & MHC class II protein complex binding & 0.001115 & 4 \\
\hline \multirow[t]{3}{*}{ MF } & GO:0023023 & MHC protein complex binding & 0.005092 & 4 \\
\hline & GO:0050786 & RAGE receptor binding & 0.006432 & 3 \\
\hline & GO:0042613 & MHC class II protein complex & 1.47E-05 & 6 \\
\hline \multirow[t]{2}{*}{ CC } & GO:0000793 & Condensed chromosome & $2.83 \mathrm{E}-05$ & 14 \\
\hline & GO:0098687 & Chromosomal region & $3.37 \mathrm{E}-05$ & 17 \\
\hline
\end{tabular}


Table 5 Gene Ontology (GO) functional enrichment analysis of candidate cell marker genes for cell type H. The top 3 GO terms in the biological process (BP), molecular function (MF) and cellular component (CC) aspects are listed (p.adjust $<0.01$ )

\begin{tabular}{|c|c|c|c|c|}
\hline Aspect & GO ID & Descriptions & p.adjust & Count \\
\hline & GO:0140014 & Mitotic nuclear division & $7.59 \mathrm{E}-06$ & 17 \\
\hline \multirow[t]{3}{*}{ BP } & GO:0019886 & $\begin{array}{l}\text { Antigen processing and presentation of exogenous peptide } \\
\text { antigen via MHC class II }\end{array}$ & 7.83E-06 & 11 \\
\hline & GO:0002495 & $\begin{array}{l}\text { Antigen processing and presentation of peptide antigen via } \\
\text { MHC class II }\end{array}$ & $8.00 E-06$ & 11 \\
\hline & GO:0023026 & MHC class II protein complex binding & 0.000107 & 5 \\
\hline \multirow[t]{3}{*}{ MF } & GO:0023023 & MHC protein complex binding & 0.000724 & 5 \\
\hline & GO:0042605 & Peptide antigen binding & 0.001458 & 5 \\
\hline & GO:0042613 & MHC class II protein complex & $6.05 \mathrm{E}-07$ & 7 \\
\hline \multirow[t]{2}{*}{ CC } & GO:0042611 & MHC protein complex & $7.81 \mathrm{E}-06$ & 7 \\
\hline & GO:0030669 & Clathrin-coated endocytic vesicle membrane & $1.06 \mathrm{E}-05$ & 8 \\
\hline
\end{tabular}

Table 6 The GO terms related to melanin and melanosomes in the GO enrichment (p.adjust< 0.05 ). "-" means that this term did not appear in the enrichment results for this cell type

\begin{tabular}{|c|c|c|c|c|}
\hline Aspects & GO ID & Descriptions & p.adjust in cell type G & $\begin{array}{l}\text { p.adjust in } \\
\text { cell type } \mathrm{H}\end{array}$ \\
\hline BP & GO:0042438 & Melanin biosynthetic process & $2.83 \mathrm{E}-05$ & 0.000325 \\
\hline BP & GO:0006582 & Melanin metabolic process & $2.83 \mathrm{E}-05$ & 0.000404 \\
\hline CC & GO:0033162 & Melanosome membrane & 4.27E-05 & 0.001092 \\
\hline CC & GO:0042470 & Melanosome & 0.000143 & 0.001458 \\
\hline BP & GO:0030318 & Melanocyte differentiation & 0.005577 & 0.049285 \\
\hline $\mathrm{BP}$ & GO:0032402 & Melanosome transport & 0.03374 & - \\
\hline $\mathrm{BP}$ & GO:0032401 & Establishment of melanosome localization & 0.03712 & - \\
\hline $\mathrm{BP}$ & GO:0032400 & Melanosome localization & 0.044045 & - \\
\hline BP & GO:0032438 & Melanosome organization & 0.044045 & 0.047491 \\
\hline
\end{tabular}

5. Comparison of the results revealed that cell type $\mathrm{G}$ was related to the mitotic process of cells, while cell type $\mathrm{H}$ was more strongly related to activities of the major histocompatibility complex (MHC).

In addition, some enriched GO terms in the two key cell types were associated with melanin and melanosomes (p.adjust $<0.05$ ), as shown in Table 6, supporting the reliability of the identification of the two key cell types.

KEGG pathway enrichment analysis of candidate cell marker genes in the two cell types are shown in Fig. 6(A) and (B). A total of 12 and 18 significantly enriched pathways (p.adjust $<0.01$ ) were related to cell types $G$ and $H$, respectively.

The two cell types shared 10 enrichment pathways, many of which were related to immune response processes, such as antigen processing and presentation, allograft rejection, and autoimmune thyroid disease. Cell type $G$ was related to DNA replication and mismatch repair pathways. Cell type $\mathrm{H}$ was related to melanogenesis and ten diseases, namely, graft-versus-host disease, rheumatoid arthritis, type I diabetes mellitus, asthma, leishmaniosis, viral myocarditis, autoimmune thyroid disease, hypertrophic cardiomyopathy, malaria, and dilated cardiomyopathy.

The functional and pathway enrichment analysis indicated that the two key cell types were strongly related to the biological activities of melanin and melanosomes. In addition, candidate cell marker genes for cell type $\mathrm{G}$ were significantly enriched in mitosis-related biological activities, and cell type $\mathrm{H}$ was associated with the occurrence of ten diseases.

\section{Discussion}

scRNA-seq technology allows researchers to study biomolecules at the single-cell level so that the molecular mechanisms of some complex diseases, such as cancer, can be studied and analyzed at a single-cell resolution.

In this paper, considering the characteristics of the scRNA-seq data, we proposed a framework for cell type identification and applied it to a single-cell melanoma dataset. Two key cell types related to melanoma were 
(A)

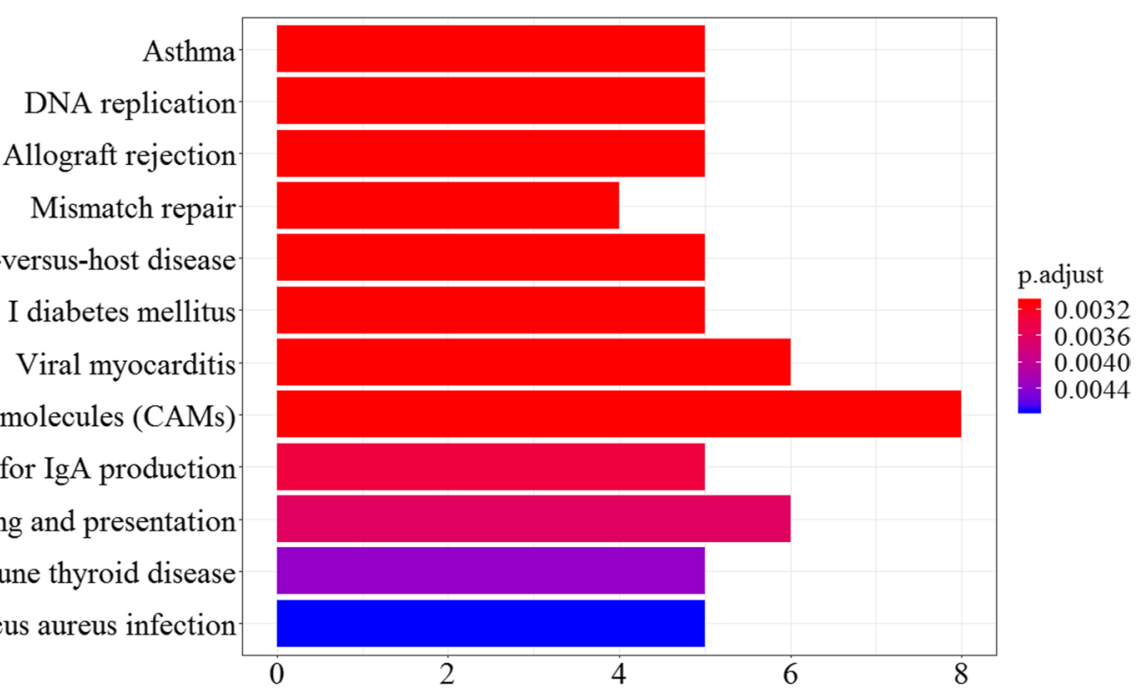

(B)

Graft-versus-host disease
twork for IgA production Rheumatoid arthritis

Type I diabetes mellitus

Asthma

Leishmaniasis

Antigen processing and presentation

Allograft rejection

Cell adhesion molecules (CAMs)

Viral myocarditis

Autoimmune thyroid disease

Staphylococcus aureus infection

Hypertrophic cardiomyopathy (HCM)

Hematopoietic cell lineage

Melanogenesis

Malaria

ECM-receptor interaction

Dilated cardiomyopathy

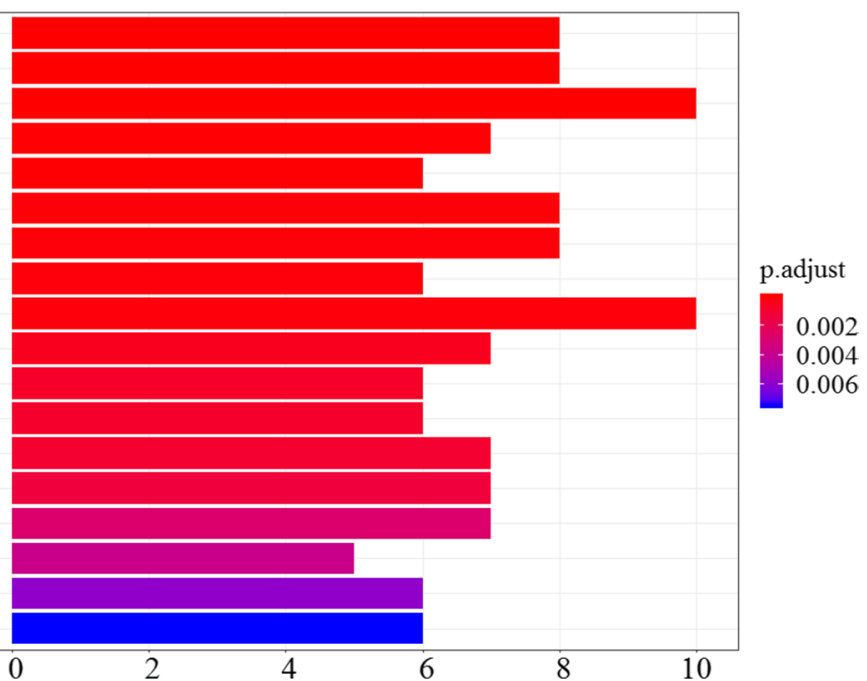

Fig. 6 KEGG pathway enrichment analysis of candidate cell marker genes for the two key cell types (p.adjust <0.01). A Cell type G. B Cell type H

identified, and the molecular mechanisms of melanoma were analyzed at the single-cell level.

First, making full use of the dropout information in the gene expression data, we identified four different dropout clusters and found that the expression levels of the protein-coding gene REXO2 differed significantly among the four dropout clusters.

Then, MCL was performed on the differential coexpression network, and 20 differential modules were identified. Then, eight cell types were identified by using the differential modules as new cell features. Our analysis identified strong correlations among cells in each cell type, with similar expression patterns, and revealed significant differences among cells of different cell types. In addition, we found that each cell type showed a different extent of association with melanoma.

Finally, we defined cell types $\mathrm{G}$ and $\mathrm{H}$ as the key cell types associated with melanoma and found that both of these key cell types were related to melanosomes and melanin and were highly correlated with the biological activities of MHC molecules. In addition, cell type $\mathrm{G}$ was related to cell mitosis, and cell type $\mathrm{H}$ was related to ten diseases.

In summary, by identifying cell types of melanoma cancer cells and further analyzing all cell types, we distinguished two key cell types that are highly related to melanoma, providing a key insight for the future direction of melanoma research. In addition, candidate cell markers for the two key cell types can be focused on as 
potential therapeutic targets for melanoma. Furthermore, the computational framework proposed in this paper is not limited to melanoma and can be extended to the pathological study of other cancers or complex diseases.

\section{Conclusion}

We proposed a computational framework for cell type identification that fully considers cell dropout characteristics. This method was applied to single-cell RNA-seq data of melanoma, and eight cell types were identified. Enrichment analysis of the candidate cell marker genes for the two key cell types showed that both key cell types were closely related to the physiological activities of the MHC. Through identification and analysis of key melanoma-related cell types, we explored the molecular mechanism of melanoma, providing insight into melanoma research. Moreover, the candidate cell markers for the two key cell types are potential therapeutic targets for melanoma.

\begin{abstract}
Abbreviations
RNA-seq: RNA sequencing; DEGs: Differentially expressed genes; scRNA-seq: Single-cell RNA sequencing; CIDR: Clustering through imputation and dimensionality reduction; MAGIC: Markov affinity-based graph imputation of cells; DBSCAN: Density-based spatial application of applications with noise; DDGs: Differentially dropout genes; DDLRs: Differentially dropout InCRNAs; DELRs: Differentially expressed IncRNAs; SCC: Spearman correlation coefficient; MCL: Markov clustering algorithm; GO: Gene ontology; KEGG: Kyoto encyclopedia of genes and genomes; BP: Biological process; MF: Molecular function; CC: Cellular component; MHC: Major histocompatibility complex.
\end{abstract}

\section{Acknowledgements}

We thank Yuhan Yang and Longting Du for helping to modify the article.

\section{About this supplement}

This article has been published as part of BMC Bioinformatics Volume 14 Supplement 5 2021: Explainable Al methods in biomedical data science (medical genomics). The full contents of the supplement are available at http://bmcme dgenomics.biomedcentral.com/articles/supplements/volume-14-suppl ement-5.

\section{Authors' contributions}

QYH and YY conceived and developed the framework for cell type identification and wrote this manuscript. LMW and GMQ provided important feedback in the framework process and edited the manuscript. FFL and YYM revised and improved the analysis process and edited the manuscript. All authors have made significant contributions to the completion and writing of this report. All authors read and approved the final manuscript.

\section{Funding}

This study was supported by the Natural Science Foundation of Shaanxi Province [No. 2017JM6038] and the National Key Research and Development Program of China [2018YFC0116500]. The publication cost for this article was funded by the National Key Research and Development Program of China [2018YFC0116500]. The funders did not play any role in the design of the study, the collection, analysis, and interpretation of data, or in writing of the manuscript.

\section{Availability of data and materials}

The data underlying this article are available in CancerSEA at http://biocc. hrbmu.edu.cn/CancerSEA/goDownload, and can be accessed with EXP0072.

\section{Declarations}

Ethics approval and consent to participate

Not applicable.

\section{Consent for publication}

Not applicable.

\section{Competing interests}

The authors declare that they have no competing interests.

Received: 11 September 2021 Accepted: 14 October 2021

Published: 17 November 2021

\section{References}

1. Situm M, Buljan M, Kolic M, Vucic M. Melanoma - clinical, dermatoscopi$\mathrm{cal}$, and histopathological morphological characteristics. Acta Dermatovener Cr. 2014;22(1):1-12.

2. Rastrelli M, Tropea S, Rossi CR, Alaibac M. Melanoma: epidemiology, risk factors, pathogenesis, diagnosis and classification. In Vivo. 2014;28(6):1005-11.

3. Barbaric J, Znaor A. Incidence and mortality trends of melanoma in Croatia. Croat Med J. 2012;53(2):135-40.

4. MacKie RM, Hauschild A, Eggermont AMM. Epidemiology of invasive cutaneous melanoma. Ann Oncol. 2009;20:1-7.

5. Schomberg J, Wang Z, Farhat A, Guo KL, Xie J, Zhou Z, et al. Luteolin inhibits melanoma growth in vitro and in vivo via regulating ECM and oncogenic pathways but not ROS. Biochem Pharmacol. 2020;177:114025.

6. Mahata P. Exploratory consensus of hierarchical clusterings for melanoma and breast cancer. IEEE Acm T Comput Bi. 2010;7(1):138-52.

7. Klinke DJ, Torang A. An unsupervised strategy for identifying epithelialmesenchymal transition state metrics in breast cancer and melanoma. Iscience. 2020;23(5):101080.

8. Jaakkola MK, Seyednasrollah F, Mehmood A, Elo LL. Comparison of methods to detect differentially expressed genes between single-cell populations. Brief Bioinform. 2017;18(5):735-43.

9. Liu KF, Ye JP, Yang Y, Shen L, Jiang H. A unified model for joint normalization and differential gene expression detection in RNA-Seq data. IEEE Acm T Comput Bi. 2019;16(2):442-54.

10. Dong M, Thennavan A, Urrutia E, Li Y, Perou CM, Zou F, et al. SCDC: bulk gene expression deconvolution by multiple single-cell RNA sequencing references. Brief Bioinform. 2020;34:1969.

11. Ziegenhain C, Vieth B, Parekh S, Reinius B, Guillaumet-Adkins A, Smets M, et al. Comparative analysis of single-cell RNA sequencing methods. Mol Cell. 2017;65(4):631-43.e4.

12. Kolodziejczyk AA, Kim JK, Svensson V, Marioni JC, Teichmann SA. The technology and biology of single-cell RNA sequencing. Mol Cell. 2015;58(4):610-20.

13. Islam S, Kjallquist U, Moliner A, Zajac P, Fan JB, Lonnerberg P, et al. Characterization of the single-cell transcriptional landscape by highly multiplex RNA-seq. Genome Res. 2011;21(7):1160-7.

14. Tang F, Barbacioru C, Wang Y, Nordman E, Lee C, Xu N, et al. mRNASeq whole-transcriptome analysis of a single cell. Nat Methods. 2009:6(5):377-82.

15. Ren X, Kang B, Zhang Z. Understanding tumor ecosystems by single-cell sequencing: promises and limitations. Genome Biol. 2018;19(1):211.

16. Zhang J, Guan M, Wang Q, Zhang J, Zhou T, Sun X. Single-cell transcriptome-based multilayer network biomarker for predicting prognosis and therapeutic response of gliomas. Brief Bioinform. 2020;21(3):1080-97.

17. Kiselev VY, Andrews TS, Hemberg M. Challenges in unsupervised clustering of single-cell RNA-seq data (vol 20, pg 273, 2019). Nat Rev Genet. 2019;20(5):310

18. Lin PJ, Troup M, Ho JWK. CIDR: Ultrafast and accurate clustering through imputation for single-cell RNA-seq data. Genome Biology. 2017;18.

19. van Dijk D, Sharma R, Nainys J, Yim K, Kathail P, Carr AJ, et al. Recovering gene interactions from single-cell data using data diffusion. Cell. 2018;174(3):716. 
20. Li WV, Li JYJ. An accurate and robust imputation method sclmpute for single-cell RNA-seq data. Nat Commun. 2018;9.

21. Yuan HT, Yan M, Zhang GX, Liu W, Deng CY, Liao GM, et al. CancerSEA: a cancer single-cell state atlas. Nucleic Acids Res. 2019;47(D1):D900-8.

22. Gerber T, Willscher E, Loeffler-Wirth H, Hopp L, Schadendorf D, Schartl M, et al. Mapping heterogeneity in patient-derived melanoma cultures by single-cell RNA-seq. Oncotarget. 2017;8(1):846-62.

23. Hamosh A, Scott AF, Amberger JS, Bocchini CA, McKusick VA. Online Mendelian Inheritance in Man (OMIM), a knowledgebase of human genes and genetic disorders. Nucleic Acids Res. 2005;33(Database issue):D514-7.

24. Sondka Z, Bamford S, Cole CG, Ward SA, Dunham I, Forbes SA. The COSMIC Cancer Gene Census: describing genetic dysfunction across all human cancers. Nat Rev Cancer. 2018;18(11):696-705.

25. Bailey MH, Tokheim C, Porta-Pardo E, Sengupta S, Bertrand D, Weerasinghe A, et al. Comprehensive characterization of cancer driver genes and mutations. Cell. 2018;174(4):1034-5.

26. Gao Y, Wang P, Wang Y, Ma X, Zhi H, Zhou D, et al. Lnc2Cancer v2.0: updated database of experimentally supported long non-coding RNAs in human cancers. Nucleic Acids Res. 2019:47(D1):D1028-D33.

27. Bao Z, Yang Z, Huang Z, Zhou Y, Cui Q, Dong D. LncRNADisease 2.0: an updated database of long non-coding RNA-associated diseases. Nucleic Acids Res. 2019;47(D1):D1034-D7.

28. Fang S, Zhang L, Guo J, Niu Y, Wu Y, Li H, et al. NONCODEV5: a comprehensive annotation database for long non-coding RNAs. Nucleic Acids Res. 2018;46(D1):D308-14.

29. Zhang XX, Lan YJ, Xu JY, Quan F, Zhao EJ, Deng CY, et al. Cell Marker: a manually curated resource of cell markers in human and mouse. Nucleic Acids Res. 2019;47(D1):D721-8.

30. Schelker M, Feau S, Du JY, Ranu N, Klipp E, MacBeath G, et al. Estimation of immune cell content in tumour tissue using single-cell RNA-seq data. Nat Commun. 2017:8(1):2032.

31. Andrews TS, Hemberg M. M3Drop: dropout-based feature selection for scRNASeq. Bioinformatics. 2019;35(16):2865-7.

32. Ester M, Kriegel H-P, Sander J, Xu X. A Density-Based Algorithm for Discovering Clusters in Large Spatial Databases with Noise. Proceedings of the Second International Conference on Knowledge Discovery and Data Mining (KDD-96); Portland, Oregon, USA. Menlo Park, California: AAAI Press; 1996. p. 226-31.

33. Zhou HB. Gao JTJAMR. Automatic Method for Determining Cluster Number Based on Silhouette Coefficient. 2014;951:227-30.

34. van Dongen $S$, Abreu-Goodger C. Using MCL to extract clusters from networks. Methods Mol Biol. 2012;804:281-95.

35. Stelzer G, Rosen N, Plaschkes I, Zimmerman S, Twik M, Fishilevich S, et al. The GeneCards Suite: From Gene Data Mining to Disease Genome Sequence Analyses. Current Protocols in Bioinformatics. 2016;54:1 30 1-1 3.

36. Ritchie ME, Phipson B, Wu D, Hu Y, Law CW, Shi W, et al. limma powers differential expression analyses for RNA-sequencing and microarray studies. Nucleic Acids Res. 2015;43(7):e47.

37. van Niel G, Charrin S, Simoes S, Romao M, Rochin L, Saftig P, et al. The tetraspanin CD63 regulates ESCRT-independent and -dependent endosomal sorting during melanogenesis. Dev Cell. 2011;21(4):708-21.

38. Berson JF, Harper DC, Tenza D, Raposo G, Marks MS. Pmel17 initiates premelanosome morphogenesis within multivesicular bodies. Mol Biol Cell. 2001;12(11):3451-64.

39. Hartman ML, Czyz M. MITF in melanoma: mechanisms behind its expression and activity. Cell Mol Life Sci. 2015;72(7):1249-60.

40. Du J, Miller AJ, Widlund HR, Horstmann MA, Ramaswamy S, Fisher DE. MLANA/MART1 and SILV/PMEL17/GP100 are transcriptionally regulated by MITF in melanocytes and melanoma. Am J Pathol. 2003;163(1):333-43.

41. Law MH, Macgregor S, Hayward NK. Melanoma genetics: recent findings take us beyond well-traveled pathways. J Invest Dermatol. 2012;132(7):1763-74.

42. Yu GC, Wang LG, Han YY, He QY. clusterProfiler: an R Package for Comparing Biological Themes Among Gene Clusters. OMICS. 2012;16(5):284-7.

\section{Publisher's Note}

Springer Nature remains neutral with regard to jurisdictional claims in published maps and institutional affiliations.
Ready to submit your research? Choose BMC and benefit from:

- fast, convenient online submission

- thorough peer review by experienced researchers in your field

- rapid publication on acceptance

- support for research data, including large and complex data types

- gold Open Access which fosters wider collaboration and increased citations

- maximum visibility for your research: over $100 \mathrm{M}$ website views per year

At BMC, research is always in progress.

Learn more biomedcentral.com/submissions 